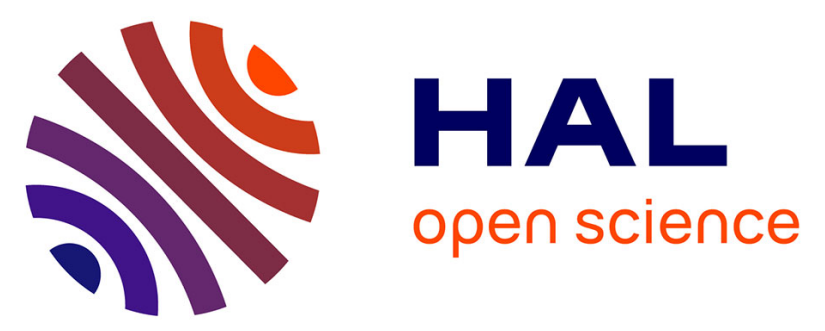

\title{
Investigation of NV (-) centers and crystallite interfaces in synthetic single-crystal and polycrystalline nanodiamonds by optical fluorescence and microwave spectroscopy
}

V. Yu. Yu Osipov, N. M Romanov, K. V Bogdanov, François Treussart, C. Jentgens, And A Rampersaud

\section{To cite this version:}

V. Yu. Yu Osipov, N. M Romanov, K. V Bogdanov, François Treussart, C. Jentgens, et al.. Investigation of $\mathrm{NV}(-)$ centers and crystallite interfaces in synthetic single-crystal and polycrystalline nanodiamonds by optical fluorescence and microwave spectroscopy. Journal of Optical Technology, 2018, 85 (2), pp.63. 10.1364/jot.85.000063 . hal-03154526

\author{
HAL Id: hal-03154526 \\ https://hal.science/hal-03154526
}

Submitted on 1 Mar 2021

HAL is a multi-disciplinary open access archive for the deposit and dissemination of scientific research documents, whether they are published or not. The documents may come from teaching and research institutions in France or abroad, or from public or private research centers.
L'archive ouverte pluridisciplinaire HAL, est destinée au dépôt et à la diffusion de documents scientifiques de niveau recherche, publiés ou non, émanant des établissements d'enseignement et de recherche français ou étrangers, des laboratoires publics ou privés. 


\title{
Investigation of $\mathrm{NV}$ centers and crystallite interfaces in synthetic single-crystal and polycrystalline nanodiamonds by optical fluorescence and microwave spectroscopy
}

\author{
V. Yu. Osipov, ${ }^{1,8}$ N. M. Romanov, ${ }^{2,3}$ K. V. Bogdanov, ${ }^{4}$ F. Treussart, ${ }^{5}$ C. Jentgens, ${ }^{6}$ and A. Rampersaud ${ }^{7}$ \\ ${ }^{1}$ A. F. Ioffe Physics and Engineering Institute, St. Petersburg, Russia \\ ${ }^{2}$ Peter the Great Polytechnic University, St. Petersburg, Russia \\ ${ }^{3}$ Lappeenranta University of Technology, Lappeenranta, Finland \\ ${ }^{4}$ ITMO University, St. Petersburg, Russia \\ ${ }^{5}$ ENS Cachan, Université Paris-Saclay, Orsay Cedex, France \\ ${ }^{6}$ Microdiamant AG, Lengwil, Switzerland \\ ${ }^{7}$ Columbus Nanoworks, Columbus, Ohio, USA \\ ${ }^{8}$ e-mail: osipov@mail.ioffe.ru
}

Three types of diamond nanoparticles with sizes from 5 to $1000 \mathrm{~nm}$ have been investigated, whose crystal lattices include nitrogen-vacancy (NV) centers: detonation nanodiamonds (DNDs), dynamic-synthesis polycrystalline diamonds, and staticsynthesis single-crystal diamonds. The electron paramagnetic resonance spectra have been investigated, along with the luminescence and IR absorption spectra of these materials. The DND concentration of $\mathrm{NV}^{-}$centers is $2.7 \mathrm{ppm}$ and is highest for particles in the size range up to $7 \mathrm{~nm}$. The concentration of $\mathrm{NV}^{-}$centers in polycrystalline diamonds is an order of magnitude less and depends on the average size of the polycrystalline particles, reaching a maximum at $180 \mathrm{~nm}$ in the average size range. The luminescence is brightest in 100-nm par-ticles of synthetic Ib diamonds subjected to high-energyelectron irradiation and annealing. The latter with an $\mathrm{NV}^{-}$concentration of around $4 \mathrm{ppm}$ can be used as fluorescent markers at the nanolevel.

\section{INTRODUCTION}

Materials that consist of nanoparticles with a crystalline surface that allows them to reliably function with various molecular agents and magnetically and optically active atomic-scale markers are currently attracting the interest of more and more researchers and developers from applied institutes and industrial companies involved in creating new engineering materials for innovative informational applications. Detonation nanodiamonds (DNDs) with a mean particle size of $5 \mathrm{~nm}$, characterized by unsurpassed chemical inertness, good biocompatibility, and the possibility of using them as a basis for creating various composite molecular systems and active molecular superstructures with predictable promising physicochemical properties are demanded as nanocrystalline precursors for a wide range of applications in engineering, material science, and biomedicine [1-3]. They were invented in the USSR, and their technological potential is not yet definitively known, because the list of possible applications is still expanding. Detonation nanodiamonds are fabricated at commercial scales by exploding a trinitrotoluol-hexogen mixture in a closed volume under conditions of negative oxygen balance, after which chemical methods are used to isolate the diamond component from the detonation mix formed in the explosion. The diamond lattice is assembled in this case under high pressures and temperatures during a time of the order of microseconds from the carbon of the explosive compounds and fragmented submolecular structures of the compounds. The nitrogen of the explosion components causes substitutional nitrogen defects to be present in the diamond lattice, while the extremely rapid assembly of the diamond lattice of the nanocrystals during the detonation synthesis causes a high concentration of defects (including vacancies and multivacancies) in the diamond lattice. The nondiamond components are removed from the mix by processing it in aqueous nitric acid at temperatures 
above $180^{\circ} \mathrm{C}$, and the diamond particles are passivated by oxygen-containing atomic groups.

Another promising diamond product, polycrystalline diamond, fabricated in the process of shock-wave loading of a powdered graphite-copper mix, during which a direct graphite-diamond phase transition occurs, also possesses a number of unique properties and is available on the market. The method of synthesizing such polycrystals was developed by Western scientists in the mid-1970s and was subsequently successfully commercialized. The graphite-containing mix is rapidly loaded and compressed by detonating an explosive charge around a thick-walled steel tube that contains this mixture (so-called DuPont technology) that is accelerated from the outside by the energy of the explosion and collapses toward its longitudinal axis, while all the nondiamond components, including the construction metal that remains after the synthesis, is eliminated by chemical means [4-6]. The polycrystals thus formed consist of very densely bonded and randomly oriented 10-15 nm crystallites of cubic diamond [7]. The unusual properties of such a diamond product include higher mechanical strength and wear resistance of the submicrometer polycrystalline diamond particles than in a bulk diamond crystal or singlecrystal diamond particles of the same size. Weak signals caused by $\mathrm{NV}^{(-)}$triplet centers were recently detected and identified by electron paramagnetic resonance (EPR) in both dynamicsynthesis materials ${ }^{1}[8]$. The specific EPR signals corresponding to $\mathrm{NV}^{(-)}$centers are caused by so-called forbidden $\Delta M_{s}=2$ optical transitions (in the microwave region) between the Zeeman-split energy levels of the triplet state of the $S=1$ center in a magnetic field $[11,12]$. It is remarkable in this case that no EPR signals corresponding to the allowed $\left(\Delta M_{s}=1\right)$ transitions are observed in triplet $\mathrm{NV}^{(-)}$centers in diamond nanoparticles with a size less than $20 \mathrm{~nm}$. It is much harder to study the luminescence of these centers, since this requires elimination of stray effects associated with potential absorption of optical radiation by carbon in the $s p^{2}$ coordination. This paper is the first (to our knowledge) to compare studies by optical and EPR methods of $\mathrm{NV}^{(-)}$centers in DNDs and shockwave-synthesized polycrystalline diamonds. In order to clarify the role of interfaces and intercrystallite boundaries in single particles or polycrystals, we study IR absorption in powders of the particles being analyzed. This is crucial because defects and intergrain boundaries that lie close to $\mathrm{NV}^{(-)}$centers can act as luminescence quenchants or can deactivate luminescence centers, including by the Förster mechanism of optical-excitation migration. The results are compared with those found for fluorescent 100-nm single-crystal nanodiamond particles obtained from microcrystals of synthetic high-pressure hightemperature (HPHT) Ib nitrogen-doped diamond by grinding, followed by irradiation with high-energy electron beams and annealing-i.e., specially fabricated samples that contain $\mathrm{NV}^{(-)}$centers.

\footnotetext{
${ }^{1}$ They are known as W15 centers in the traditional classification of paramagnetic defects in diamond. The W15 center was first observed in electron- or proton-irradiated type Ib diamonds by South African scientists (Loubser and Van Wyk) in 1977 [9]. The electron-level structure of this center was subsequently studied and described in detail in [10].
}

\section{SAMPLES, MATERIAL-SYNTHESIS TECHNOLOGY, AND RESEARCH METHODS}

This project involved fabricating and processing three different groups of nanodiamond materials with $\mathrm{NV}^{(-)}$centers unintentionally created in the material at the synthesis stage or created after synthesizing the crystallites but now on purpose. The processes had the purpose of promoting either the appearance of luminescence from the $\mathrm{NV}^{(-)}$centers or the detection of specific EPR signals from the $\mathrm{NV}^{(-)}$centers on the background of broad EPR signals from iron-containing complexes and compounds.

Different fractions of polycrystalline diamond powders from Van Moppes \& Sons (Geneva, Switzerland) with a mean size that varied from 25 to $1000 \mathrm{~nm}$ were selected as the basic test material. Differently sized fractions were obtained by grinding large polycrystalline diamond particles with a size from 10 to $50 \mu \mathrm{m}$, produced using the DuPont technology, and then isolating a fraction of the particles with a mean size of $2-3 \mu \mathrm{m}$, after which the particles of this fraction were separated into submicrometer fractions by sedimentation in an aqueous medium and high-speed centrifugation.

Powders of the fine fractions were given additional chemical purification in mixtures of hot acids, using the equipment ${ }^{2}$ of the Microdiamant Co. (Lengwill, Switzerland) and ENS Cachan (Université Paris-Saclay, France), in order to remove the graphite-like light-absorbing $s p^{2}$ phase around the polycrystalline particles and the $3 d$ metallic impurities, mainly iron, that got into the material at the synthesis stage. The purity was monitored by $\mathrm{x}$-ray fluorescence analysis. The purity was also monitored from the absence of a broad EPR signal from iron-containing complexes and iron compounds in the magnetic-field region corresponding to EPR signals with $g$-factor 4 . The latter is a necessary but not sufficient condition for recording weak EPR signals with $g$-factor $g=4.26$ from $\mathrm{NV}^{(-)}$centers. Lyophilic powders of gray color are obtained as a result of hot acid processing. The polycrystalline diamond powders purified in acids are designated in what follows as PCD- $i$, where index $i$ (from 1 to 9) corresponds to fractions of different size ranges. Data on the different fractions of the PCD powders are indicated in Table 1.

The chosen comparison sample was DND powder produced by Special Technical Design Office Tekhnolog (St. Petersburg, Russia) and processed with aqueous nitric acid in an autoclave at $220^{\circ} \mathrm{C}-235^{\circ} \mathrm{C}$, repeatedly rinsed in de-ionized water, and then dried. Primary drying was carried out at a temperature (no greater than $350^{\circ} \mathrm{C}$ ) high enough to remove sorbed molecular groups but insufficient to gasify the carboncontaining fragments from the surface. This material, with a surface precoated with oxygenated groups, is then designated as DND-ox. The graphite-like phase in DND-ox was removed by oxidizing it and gas-phase etching in an oxygen-containing air atmosphere in a laboratory furnace at a fixed temperature chosen in the range from $420^{\circ} \mathrm{C}$ to $480^{\circ} \mathrm{C}$ for $12 \mathrm{~h}$. About $50 \mathrm{mg}$ of the DND-ox powder was held in the furnace during each heat treatment. The fall-off of the mass at temperatures

\footnotetext{
${ }^{2}$ Besides general-purpose chemical equipment, laboratory autoclaves with mi-
} crowave heating (Anton Paar GmbH, Germany) were used. 
Table 1. Particle and Crystallite Sizes in Different Powder Fractions of Single-Crystal and Polycrystalline Nanodiamonds

\begin{tabular}{lcc}
$\begin{array}{l}\text { Marking of } \\
\text { Nanodiamond } \\
\text { Powders }\end{array}$ & $\begin{array}{c}\text { Range of } \\
\text { Particle } \\
\text { Sizes, } \boldsymbol{\mu m}\end{array}$ & $\begin{array}{c}\text { Mean Size of Crystallites } \\
\text { and/or Polycrystalline } \\
\text { Aggregates in Powder, nm }\end{array}$ \\
\hline $\begin{array}{l}\text { Polycrystalline diamond } \\
\text { PCD-1 }\end{array}$ & $0-0.05$ & $25^{a}(7-9)^{b}$ \\
PCD-2 & $0-0.10$ & $50^{a}(7-9)^{b}$ \\
PCD-3 & $0-0.15$ & $75^{a}(7-9)^{b}$ \\
PCD-4 & $0-0.20$ & $90^{a}(7-9)^{b}$ \\
PCD-5 & $0-0.35$ & $180^{a}(7-9)^{b}$ \\
PCD-6 & $0-0.50$ & $230^{a}(7-9)^{b}$ \\
PCD-7 & $0.25-0.50$ & $350^{a}(7-9)^{b}$ \\
PCD-8 & $0.5-1.00$ & $750^{a}(7-9)^{b}$ \\
PCD-9 & $0.75-1.25$ & $1000^{a}(7-9)^{b}$ \\
Detonation diamond & & \\
DND & $\leq 0.050$ & $4.5^{b}$ \\
Synthetic HPHT Ib & & \\
diamond & & $100^{c}$ \\
FND-1 & $0-0.20$ & $100^{c}$ \\
FND-2 & $0-0.20$ &
\end{tabular}

${ }^{a}$ Manufacturer's data: for all fractions, the width of the particle-distribution function over sizes is of the order of the mean size of the polycrystalline particles.

${ }^{b}$ Size of the $\mathrm{x}$-ray coherent-scattering region.

'Scanning-electron-microscope data for the crystallites.

above $480^{\circ} \mathrm{C}$ (for the $\mathrm{DND}$-ox) was recorded, and the powder remaining after this processing was studied by IR spectroscopy.

Particles of fluorescent nanodiamonds (FNDs) with a mean size of $100 \mathrm{~nm}$ were used as material with specially induced $\mathrm{NV}^{(-)}$centers. These were obtained from synthetic (with atomic nitrogen impurities) microcrystalline HPHT Ib diamond with grain size $150 \mu \mathrm{m}$. The latter was first subjected to intense comminution to the submicrometer level, and the isolated superfine fraction was then irradiated in a thin layer with a beam of high-energy $(5-\mathrm{MeV})$ electrons and was annealed in an inert atmosphere at $800^{\circ} \mathrm{C}$. Here high-energy irradiation was used to create vacancies in the diamond lattice, and the subsequent annealing caused the vacancies to diffuse in the lattice and caused them to be captured on isolated nitrogen impurities (C defects). The nitrogen concentration in the microcrystalprecursors was $150 \mathrm{ppm}$ (parts per million). The current in the electron beam was $25 \mathrm{~mA}$. The minimum irradiation dose was $7.0 \times 10^{18}$ electrons $/ \mathrm{cm}^{2}$. The electron-irradiated materials with exposures that differed by a factor of 2 ( 16 and $32 \mathrm{~h}$ ) were then designated as FND-1 and FND-2. A fraction with a mean size of $100 \mathrm{~nm}$ was extracted using centrifugation in an aqueous medium. The FND powders were fabricated and supplied by Columbus Nanoworks Co. (Columbus, Ohio, USA).

Table 1 shows the marking of all the test samples of powders of polycrystalline, detonation, and ground single-crystal diamonds corresponding to the mean size of the individual particles and the ranges of variation of particle sizes in these powders.

The EPR spectra of the samples were recorded at room temperature in the X-range by means of an Elexsys E-680X Bruker EPR spectrometer at a frequency of $9.4 \mathrm{GHz}$. A quantity of $50-60 \mathrm{mg}$ of the powder was poured into a clear fused quartz
(CFQ) tube (Wilmad quartz EPR tubes, Sigma-Aldrich) $4 \mathrm{~mm}$ in outer diameter, having a powder column in the tube no more than $12 \mathrm{~mm}$ high. The open end of the tube was sealed against moisture. The tube with powder was fixed in the chamber of the microwave cavity. Spectra of EPR signals with $g$-factors in the range 4.00-4.30 were recorded at a microwave radiation power in the range $0.2-2 \mathrm{~mW}$, modulation amplitude $3-10 \mathrm{G}$, magnetic-field modulation frequency $100 \mathrm{kHz}$, number of signal accumulations $1-50$, chosen for weak signals from the optimum SNR conditions for recording. The time constant for one discrete reading was $0.020 \mathrm{~s}$, and the total time was $100 \mathrm{~s}$ to record the spectrum for one pass in the magnetic-field sweep interval from 1300 to $2000 \mathrm{G}$. At microwave powers $P_{\mathrm{MW}}<4 \mathrm{~mW}$, the intensity of an EPR signal with a $g$-factor of $g \approx 4$ followed the linear dependence $I_{\mathrm{pp}} \approx\left(P_{\mathrm{MW}}\right)^{1 / 2}$ in the detonation and polycrystalline diamonds, and no signal-shape distortions from saturation were recorded. The concentrations of paramagnetic $\mathrm{NV}^{(-)}$centers in the test samples were estimated by double integration of the corresponding EPR signal $(g=4.26)$ and the ratio of the measured value to the weight of the sample. Detonation nanodiamond powder with a known concentration of paramagnetic $S=1 / 2$ centers equal to $6.3 \times$ $10^{19} \mathrm{~g}^{-1}$ was used as a standard [13].

The secondary-emission spectra, which consists of luminescence radiation, along with Raman scattering, were recorded by means of an inVia $\mu$-Raman apparatus (Renishaw, UK), using exciting laser radiation at a wavelength of $532 \mathrm{~nm}(\text { or } 488 \mathrm{~nm})^{3}$ and a $50 \times$ microscope lens that focuses the radiation into a spot about $2 \mu \mathrm{m}$ in diameter on the surface of the sample. The secondary radiation was collected from the surface of the sample in the reverse-scattering geometry. A sample was fabricated for luminescence studies by pressing the powder into a cylinder $3 \mathrm{~mm}$ in diameter and no more than $2 \mathrm{~mm}$ thick inside a metallic holder. Fluorescence images of the brightest particles deposited from a droplet of the aqueous suspension were obtained by means of a Nikon Eclipse TiS epifluorescence inverted microscope using a Texas Red Cube standard optical filter (excitation 540-580 nm, transmitting 600-660-nm radiation), which transmits the main part of the emission from the $\mathrm{NV}^{(-)}$centers. The images are obtained with a $10 \mathrm{~ms}$ exposure by means of a $10 \times$ microscope lens.

Optical transmission spectra in the $700-4000 \mathrm{~cm}^{-1}$ region were recorded by means of a Specord-M80 two-beam IR spectrophotometer (Carl Zeiss, Jena, Germany). Powder of the test substance was pressed into a matrix of Uvasol spectroscopic-quality potassium bromide (CAS\# 7758-02-3) (Merck, Darmstadt, Germany) under forevacuum pumping conditions. The weight concentration of the test substance in the dry mixture used for pressing was about 1:1000 or 4:1000 and was selected to optimize the modulation of the bands and lines with the highest absorption on the transmission spectra (dips all the way to $30 \%-50 \%$ of the maximum transmission level of the matrix). The spectra were recorded with a 2 or $4 \mathrm{~cm}^{-1}$ step. The time constant was chosen to equal 30 or 60 s. To obtain

\footnotetext{
${ }^{3}$ Exciting radiation with wavelength $488 \mathrm{~nm}$ was used only when studying the secondary-emission spectra of nanoparticles of polycrystalline diamond PCD, where the number of observable luminescence bands reached four or five.
} 
(a)

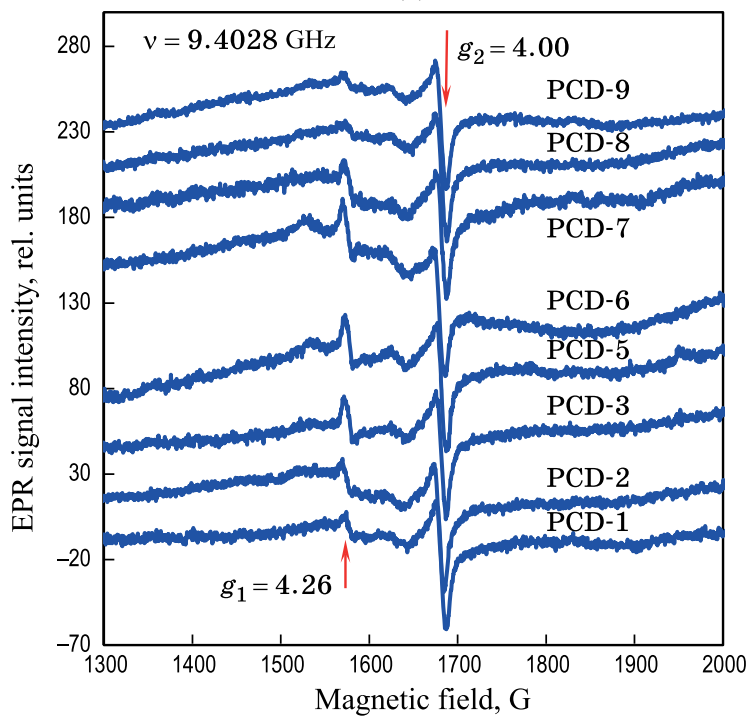

(b)

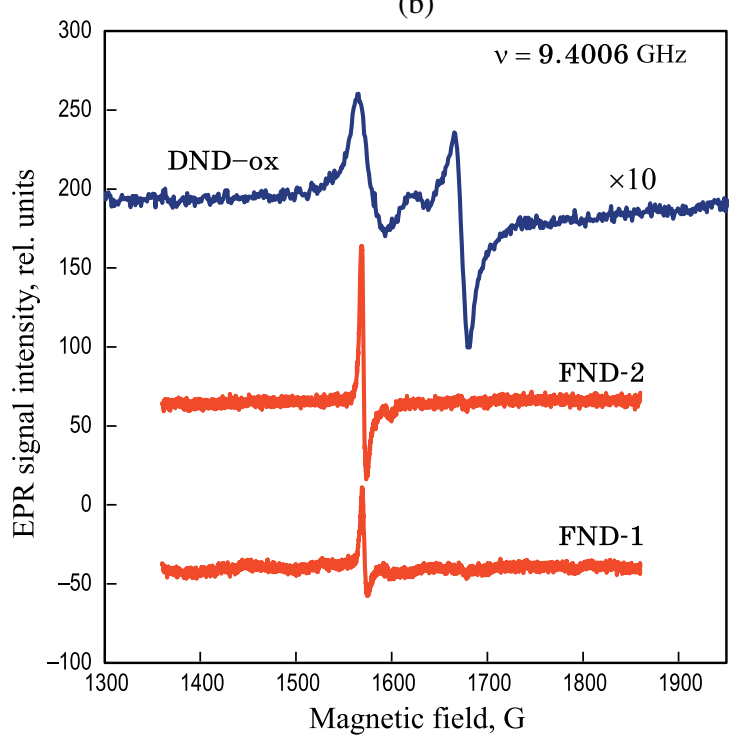

Fig. 1. (a) EPR spectra of different size-fractions (PCD-1-PCD-9) of dynamic-synthesis polycrystalline diamond and (b) detonation nanodiamond DND-ox and fluorescent synthetic HPHT Ib diamond (FND-1, FND-2) with mean particle size $100 \mathrm{~nm}$. The sizefractions of the PCD polycrystals are designated on each spectrum in (a). The spectra in (a) are normalized to the intensity of the high-field EPR line with $g$-factor $g_{2}=4.00$ (from multivacancies). The microwave frequency is $9.40 \mathrm{GHz}$.

the absorption spectra of the test material, the resulting experimental data were corrected to take into account the spectral transmission of a pressed pellet made from pure $\mathrm{KBr}$ and were recalculated.

\section{EXPERIMENTAL RESULTS AND DISCUSSION}

\section{A. Microwave Spectroscopy}

The EPR spectra of different size-fractions of polycrystalline diamond (PCD) and DND-ox powder are shown in Figs. 1(a) (a)

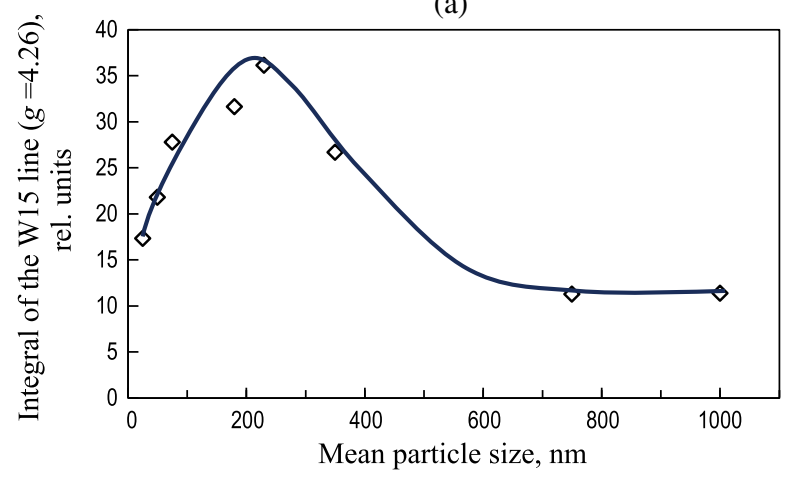

(b)

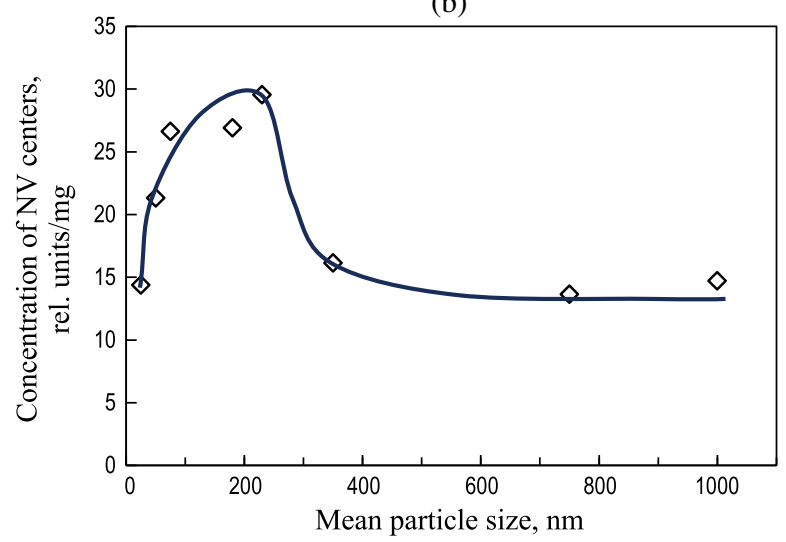

Fig. 2. (a) Peak intensity of the EPR line of the $\mathrm{NV}^{(-)}$centers with $g$-factor $g_{1}=4.26$ normalized to the intensity of the EPR line with $g$-factor $g_{2}=4.00$ and (b) the weight concentration of the $\mathrm{NV}^{(-)}$centers in the diamond polycrystals versus the mean size of the polycrystalline diamond aggregate.

and $1(\mathrm{~b})$ in the magnetic-field range from 1300 to $2000 \mathrm{G}$. The low-field EPR line with $g$-factor $g_{1}=4.26$ corresponds to $\mathrm{NV}^{(-)}$centers, while the high-field line ${ }^{4}$ with $g$-factor $g_{2}=$ 4.00 corresponds to the multivacancies formed in the crystals at the dynamic-synthesis stage as a result of the aggregation of vacancies into linear clusters of small extent. The intensities of both EPR lines in the DNDs $\left(g_{1}=4.26\right.$ and $\left.g_{2}=4.00\right)$ are about equal, and the lines themselves are relatively broad. They were first recorded 10-12 years ago [13] in the EPR spectra of DNDs thoroughly purified from $3 d$ metals and were subsequently identified as forbidden $\left(\Delta M_{s}=2\right)$ microwavefield-induced transitions between Zeeman-split energy levels of the triplet state of centers with spin $S=1$ [8]. The specific location of the lines on the magnetic-field scale and the features of their shape are determined by the individual parameters of the spin Hamiltonian, which describes the exchange interaction of individual spins of $1 / 2$ within a triplet center of each type $\left(\mathrm{NV}^{(-)}\right.$and multivacancy) in the crystal lattice [8]. As can be seen from Fig. 1(a), the intensity of the $g_{1}=$ 4.26 line strongly depends on the mean size of the polycrystals,

${ }^{4}$ This line is separated from the low-field line by about $100 \mathrm{G}$ on the magneticfield scale and is clearly identified on the EPR spectra of not only all the sizefractions of the polycrystalline diamonds, but also of the DNDs. 
whereas the overall ascending background and the intensity of the $g_{2}=4.00$ line show virtually no change in the series of PCD test samples. The corresponding size dependence of the intensity ${ }^{5}$ of the $g_{1}=4.26$ line is shown in Fig. 2(a). It displays a pronounced maximum in the mid-range of sizes and a sharp falloff in the region above $350 \mathrm{~nm}$. The weight concentration of $\mathrm{NV}^{(-)}$centers can be estimated by double integration of this line contour or as a product of the square of the line width $\Delta H_{p p}$ times its amplitude $I_{p p}$, after which this parameter is referred to as the mass of the powder. The spectra shown in Fig. 1(a) allow the $g_{1}=4.26$ lines of different amplitudes to be processed in this way. The concentration of $\mathrm{NV}^{(-)}$centers increases approximately linearly as the mean size of the polycrystals increases from 25 to $90 \mathrm{~nm}$ and reaches a maximum at $180-230 \mathrm{~nm}$, after which it strongly falls off in the interval to $1000 \mathrm{~nm}$ [Fig. 2(b)]. The concentration of $\mathrm{NV}^{(-)}$centers is about $0.3 \mathrm{ppm}$ at the maximum (for the PCD-6 fraction). The multivacancy concentration, determined by double integration of the high-field EPR line with $g$-factor $g_{2}=4.00$, remains approximately constant and shows virtually no dependence on the size of the polycrystals in the interval from 25 to $1000 \mathrm{~nm}$. Such an observation was also pointed out in [14]. This can be indirect evidence not only of a reduction of the fraction of $\mathrm{NV}^{(-)}$centers in the material when there is a transition to small particles in connection with the breakdown of the centers in mechanically damaged regions of the lattice close to the outer surface but also of enrichment of the polycrystalline particles with nitrogen and not with vacancies for fractions of particles with a mean size in the range from 25 to $180 \mathrm{~nm}$, possibly caused by the morphological features of the formation of large polycrystalline aggregates during shock-wave loading, after which they are separated into submicrometer fractions. The sections of the lattice that contain more impurity nitrogen are usually less mechanically strong and easily split apart from larger particles during grinding or even break down into still finer fragments. The width of the EPR lines $\left(g_{1}=4.26\right)$ for $\mathrm{NV}^{(-)}$centers in polycrystals of all the size-fractions PCD-1 to PCD-9 are about the same, but less than for DND-ox. The $g_{1}=4.26$ line is about $13 \mathrm{G}$ wide in DND-ox, while it is about $8-9 \mathrm{G}$ wide in PCD. (The EPR high-field $g_{2}=4.00$ line is also about $8-9$ G wide in PCD powders and is virtually independent of the mean size of the polycrystals.) On the assumption that the $g_{1}=4.26$ EPR line undergoes dipole-dipole or exchange broadening, this indicates a substantially larger spacing from the isolated $\mathrm{NV}^{(-)}$ centers with spin $S=1$ in the diamond lattice to the closest paramagnetic $S=1 / 2$ centers (broadening agents) at the intergrain boundaries and/or the crystallite interfaces in PCD than in DND-ox. This is very reasonable, taking into account that the mean crystallite size equals $10-15 \mathrm{~nm}$ in PCD and $4.5 \mathrm{~nm}$ in DND-ox. The probable conditions for luminescence quenching thus look much less rigorous in PCD than in DNDox, where the crystallite boundary gets much closer to the radiative luminescence center inside the nanoparticle. The

\footnotetext{
${ }^{5}$ The peak intensity $I_{p p}$-i.e., the distance between the maximum and minimum along the vertical on the spectrum of the first derivative-is implied from the microwave absorption.
}

concentration of $\mathrm{NV}^{(-)}$centers in DND-ox in this case is estimated as approximately $2.7 \mathrm{ppm}$, which is more than sufficient in practice to obtain bright luminescence from the nanoparticles, provided luminescence-quenching processes are neglected. There is one $\mathrm{NV}^{(-)}$center for thirty $5 \mathrm{~nm}$ DND particles in this case, and this is insufficient for the individual 5-nm particles to be used as separate luminescence markers. However, the 30-35 nm DND aggregates that are most stable in aqueous solutions and which consist of more than a hundred individual nanoparticles always contain several $\mathrm{NV}^{(-)}$centers and are suitable for marking at the submicrometer level.

The EPR spectra of the FND-1 and FND-2 samples with an irradiation dose by high-energy electrons that differs by about a factor of 2 are shown in Fig. 1(b). It can be seen that, in a magnetic field that is half as strong, ${ }^{6}$ the spectra contain only the characteristic narrow $g_{1}=4.26$ line from $\mathrm{NV}^{(-)}$centers of different amplitudes, approximately proportional to the irradiation dose, but there are no signals from multivacancies or other triplet centers [Fig. 1(b)]. The EPR signal with $g$-factor $g_{1}=4.26$ (from the $\mathrm{NV}^{(-)}$centers) is absent from the original nonirradiated HPHT Ib crystal in this case, and this is evidence that $\mathrm{NV}^{(-)}$centers appear in the crystals only after they are irradiated with high-energy electrons and annealed. At the same time, unlike the DND and PCD materials with small crystallites (about 5 and 10-15 nm across), the FND-1 and FND-2 EPR spectra demonstrate a strong signal from P1 centers (substitutional nitrogen) in the 3240-3460-G magnetic-field region with resolved superfine structure, associated with magnetic interaction of the unpaired antibonding orbitals of the P1 center with the nuclear magnetic moment of the nitrogen atom. ${ }^{7}$ The central $g=2.0024$ line, with symmetric low- and high-field satellites separated by $60 \mathrm{G}$ on the magnetic-field scale, is the characteristic signature of P1 centers. This is evidence of the high quality of the specially fabricated FND particles with substitutional nitrogen impurities in which the concentration of all the paramagnetic defects with spin $1 / 2$ does not exceed $130 \mathrm{ppm}$. At the same time, an analysis of the overall intensity of the $g_{1}=4.26$ line is evidence that the concentration of fluorescent $\mathrm{NV}^{(-)}$centers in FND-2 is about $4 \mathrm{ppm}$. The EPR method can thus be used for an independent estimate of the concentration of $\mathrm{NV}^{(-)}$centers in fluorescent diamonds smaller than $100-150 \mathrm{~nm}$, where these centers were unintentionally created (during synthesis) or specially created (by electron-beam irradiation after synthesis).

\section{B. Luminescence Properties}

The secondary-emission spectra of polycrystalline diamonds PCD-2, PCD-4, and PCD-5 with a mean polycrystal size of 50, 90, and $180 \mathrm{~nm}$ and DND-ox powder processed in air at $430^{\circ} \mathrm{C}$ are shown in Figs. 3(a) and 3(b). The DND-ox luminescence spectrum with a maximum at $678 \mathrm{~nm}$ is

\footnotetext{
${ }^{6}$ This refers to a magnetic-field region in which there are weak EPR signals with a $g$-factor of $g \approx 4$ and its neighborhood. This actually occurs in magnetic fields that are half as great as those in which the main EPR signal is observed for nanodiamonds with a $g$-factor of $g=2.0024-2.0027$.

${ }^{7}$ The EPR spectrum of this signal in the $3240-3460-G$ region is not shown here, since the form of the signal is standard, with typical triplet superfine structure.
} 
(a)

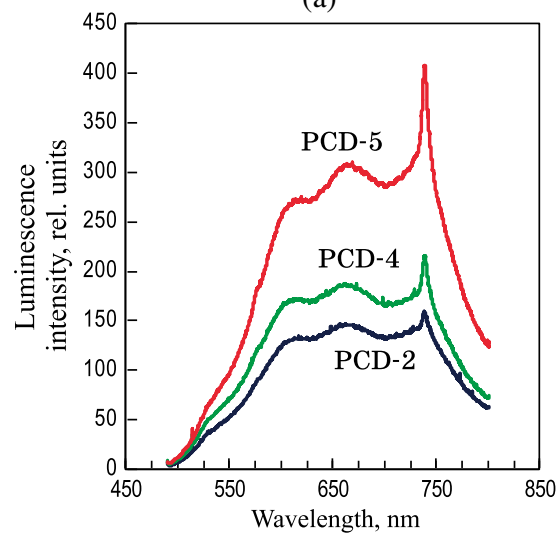

(b)

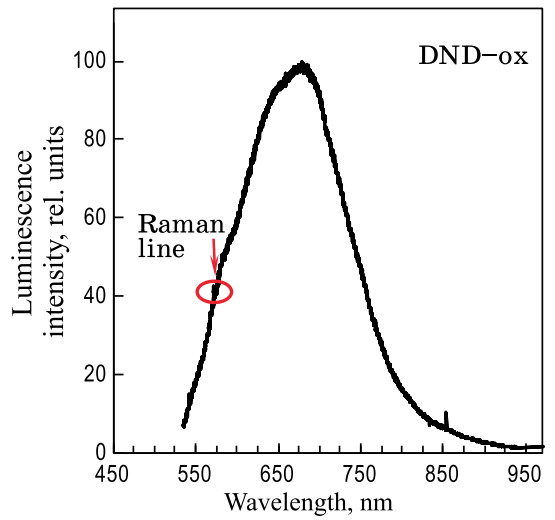

(c)

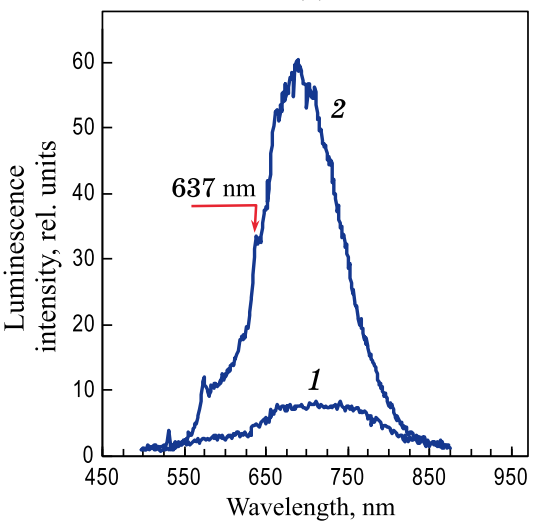

Fig. 3. Secondary-emission spectra of nanodiamond particles with $\mathrm{NV}^{(-)}$centers in materials of different synthesis and processing types. (a) Polycrystalline diamond (PCD-2, PCD-4, PCD-5) with a mean aggregate size of 50, 90, and 180 nm; (b) DND-ox; and (c) FND-1 (1) and FND-2 (2). The spectra were excited by laser radiation at (a) $488 \mathrm{~nm}$ and/or at (b), (c) $532 \mathrm{~nm}$.

predominantly associated with the radiation of $\mathrm{NV}^{(-)}$centers [Fig. 3(b)]. The wide emission band that stretches to $770 \mathrm{~nm}$ or further is associated with multiple (usually up to eight) phonon sidebands [10], as occurs in micrometer-size single crystals. The background band in this case is $700 \mathrm{meV}$ wide on the energy scale [10] (at the 5\% level of the maximum intensity). Since the mean DND-ox particle size is $4-5 \mathrm{~nm}$, and the phononconfinement effect is substantial under these conditions, the fine structure of the phonon wing of the emission spectrum (with several characteristic peaks) is not spectrally resolved. The position of the Raman line of diamond ${ }^{8}$ in DND-ox particles is shifted by $8-10 \mathrm{~cm}^{-1}$ relative to the corresponding value for bulk diamond $\left(1332 \mathrm{~cm}^{-1}\right)$ because of the phononconfinement effect and equals $1324 \mathrm{~cm}^{-1}$, while the line width at half-height is about $25 \mathrm{~cm}^{-1}$. In turn, the luminescence spectra of very different fractions of polycrystalline diamonds (PCD-2, PCD-4, and PCD-5) demonstrate several characteristic bands (up to four or five) that are easily separated and identified by resolving the spectra into Gaussian contours or mixed Gaussian-Lorentzian types [Fig. 3(a)]. One of these, a narrow long-wavelength band centered at $739 \mathrm{~nm}$, is associated with $\mathrm{SiV}$ centers, while another, moderately wide band with a maximum at $663 \mathrm{~nm}$ is associated with $\mathrm{NV}^{(-)}$centers. The latter attribution is based on the fact that EPR spectroscopy shows that an appreciable number of $\mathrm{NV}^{(-)}$centers are present in the material. One more wide photoluminescence band is the most intense band and lies in the short-wavelength region, with its maximum at $610 \mathrm{~nm}$; it may be associated with emission from $\mathrm{NV}^{(0)}$ centers. The green luminescence band with maximum at $525 \mathrm{~nm}$ is most likely associated with emission from NVN (or H3) centers. These bands were first detected and some of them (at $739 \mathrm{~nm}$ ) were described in detail in [15]. The intensities of both red luminescence bands may be directly associated with the number of NV centers in neutral and negatively charged states, the latter of which are easy to detect by means of EPR. For polycrystal particles with a mean

\footnotetext{
${ }^{8}$ The position of the Raman line of diamond is denoted by an arrow on the secondary-emission spectrum in Fig. 3(b).
}

size in the range from 25 to $230 \mathrm{~nm}$, good correlation is observed between two of these parameters - the luminescence intensity in the 600-750 $\mathrm{nm}$ band and the $\mathrm{NV}^{(-)}$concentration determined by EPR. The red luminescence band is most intense for polycrystal fractions with a mean size of $180 \mathrm{~nm}$ and/or $230 \mathrm{~nm}$.

The secondary-emission spectra of FND-1 and FND-2 obtained with excitation by $532 \mathrm{~nm}$ laser radiation demonstrate characteristic wide luminescence bands associated with emission from $\mathrm{NV}^{(-)}$defects [Fig. 3(c), lower (1) and upper (2) spectra]. In this case, for material with a short exposure time by high-energy electrons (FND-1) and a correspondingly low concentration of $\mathrm{NV}^{(-)}$defects, the luminescence band has virtually no structure in the interval from 600 to $820 \mathrm{~nm}$. In turn, the material with twice the exposure time (FND-2) and a larger concentration of $\mathrm{NV}^{(-)}$defects demonstrates fine features in the luminescence spectra, including a zero-phonon line at $637 \mathrm{~nm}$ and a band in the $650-820 \mathrm{~nm}$ range associated with phonon side bands. The luminescence intensity of FND-2 in this case turns out to be a factor of 7-8 higher than that of FND-1. The fluorescence image of a dried droplet of a suspension of FND-2 nanoparticles obtained using the Texas Red Cube filter, which transmits radiation in the $600-660 \mathrm{~nm}$ interval, is shown in Fig. 4. It has the shape of a narrow luminous red ring, where the precipitated material formed during the specific drying of the water droplet has its maximum concentration. The superior fluorescence characteristics of FND-2 have two simultaneous causes - a high concentration of artificially created $\mathrm{NV}^{(-)}$centers, which reaches $4-5 \mathrm{ppm}$, and high optical quality of the diamond lattice in individual particlecrystals, with a mean size of $100 \mathrm{~nm}$ obtained by grinding. The number of defects induced by grinding in the lattice and in the mechanically disturbed region close to the surface of the particles is negligible for quenching the luminescence from the overwhelming number of $\mathrm{NV}^{(-)}$centers inside such $100 \mathrm{~nm}$ particles. The fluorescence characteristics (spectral composition, luminescence intensity) are about the same in specially irradiated and annealed synthetic micrometer-size HPHT crystals of Ib diamond [16]. 
(a)
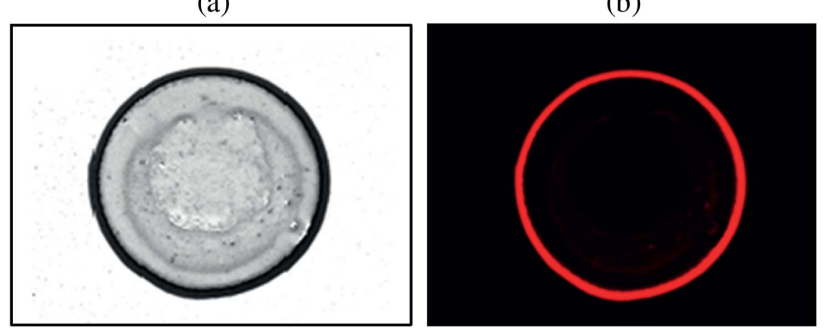

Fig. 4. (a) Optical image of a dried drop of an aqueous suspension of FND-2 synthetic diamond nanoparticles with $\mathrm{NV}^{(-)}$centers and (b) the corresponding fluorescence image of the drop, obtained using an epifluorescence microscope, a Texas Red Cube red filter (600$660 \mathrm{~nm}$ ), and an illuminating laser with wavelength $532 \mathrm{~nm}$. The observed red ring corresponds to the sections on the substrate in which the concentration of precipitated FND-2 particles is greatest when the sample dries out. The ring is $0.8-0.9 \mathrm{~mm}$ in diameter.

In the PCD polycrystals studied in this paper, the $\mathrm{NV}^{(-)}$ centers are located in 10-15 $\mathrm{nm}$ crystals, with several (as many as three or four) crystallites on the average for every $\mathrm{NV}^{(-)}$ center. A model of polycrystals with $\mathrm{NV}^{(-)}$centers is shown in Fig. 5(a). The $\mathrm{NV}^{(-)}$centers close to the outer boundaries of the polycrystals are more favorably located in principle from the viewpoint of emitting radiation than the centers deep inside large polycrystals. However, effects induced by grinding and cracking the original large polycrystalline particles along intergrain boundaries can also deactivate the $\mathrm{NV}^{(-)}$centers that lie close to the outer surfaces of the polycrystals, including by reversing their electrical charge or even by destroying them. This may have the result that the weight concentration of $\mathrm{NV}^{(-)}$centers smoothly decreases as the average particle size decreases from 180 to $25 \mathrm{~nm}$ according to the EPR spectroscopy data.

It is clear that high luminescence output from materials that contain $\mathrm{NV}^{(-)}$centers can be ensured by reducing the absorption of the radiation emitted at the surfaces and grain boundaries of the particles, particularly those that contain graphene-like $s p^{2}$ fragments of various thicknesses. The presence of $s p^{2}$-hybridized carbon in PCD polycrystals was demonstrated by Raman spectroscopy [15] and is also confirmed (a)

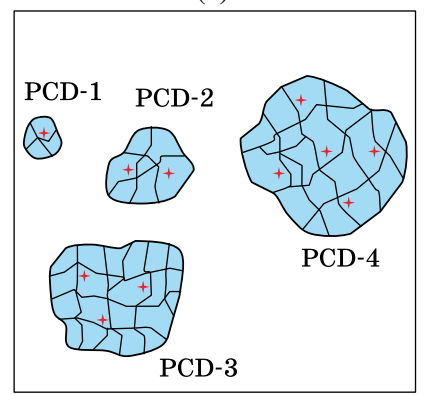

(b)

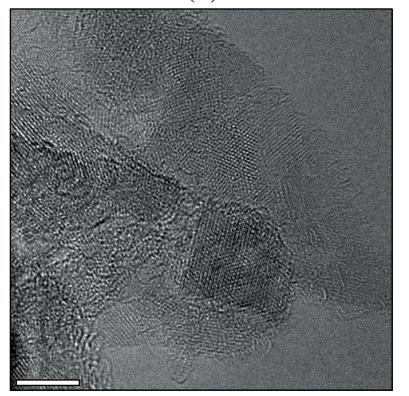

Fig. 5. (a) Diagram of the structure of polycrystalline diamond particles of different size-fractions and (b) an image of PCD-1 polycrystals with mean size $25 \mathrm{~nm}$, obtained by high-resolution transmission electron microscopy. The red crosses schematically show the positions of the $\mathrm{NV}^{(-)}$centers in the individual crystallites of the polycrystal. The scale strip on the right-hand panel is $4 \mathrm{~nm}$ wide. by high-resolution transmission electron microscopy. One way to eliminate graphene-like fragments from the crystallite surface is by selective liquid-phase (in acids at high temperatures) or gas-phase oxidation of the surface, so that easily oxidized components are eliminated in the liquid-phase or gas-phase processes. Many materials, including DND-ox and PCD, become luminescent as a result of such auxiliary processing. We shall consider the IR absorption characteristics of materials that undergo such oxidative processing.

\section{Infrared Absorption}

Figure 6(a) (curve 1) shows the IR absorption spectrum of sample PCD-1 after final processing in mixtures of acids. This spectrum contains a broad structureless band in the $1000-1300 \mathrm{~cm}^{-1}$ interval, predominantly associated with vibrational absorption of $\mathrm{C}-\mathrm{O}$ and $\mathrm{C}-\mathrm{O}-\mathrm{C}$ groups on the surfaces of the crystallites that compose the polycrystals and two narrow bands in the neighborhood of $1630 \mathrm{~cm}^{-1}$ and $1800 \mathrm{~cm}^{-1}$, associated with the absorption and valence vibrations of hydroxyl and carbonyl groups $[17,18]$. A pronounced band from the carbonyl groups appears at $1800 \mathrm{~cm}^{-1}$ in the absorption spectra of the processed material [Fig. 6(a), curves 1-4] as a result of acid processing of the polycrystalline PCD samples of all the smallest size-fractions (less than $200 \mathrm{~nm}$ ) in an autoclave. However, all the features in the $900-1500 \mathrm{~cm}^{-1}$ interval of the wide absorption band of the various fractions of the materials subjected to acid processing are maintained after processing - even the details of the bands at 1100 and $1200 \mathrm{~cm}^{-1}$. This means that the primary functional groups that appear on the inner and outer interfaces of the polycrystals at the synthesis and extraction stages are inert and stable against chemical processing in mixtures of acids in an autoclave at $170^{\circ} \mathrm{C}$. The absorption band at $1100-1110 \mathrm{~cm}^{-1}$ is present on the IR spectra of the PCD-1-PCD-4 samples of all the smallest size-fractions obtained by separation and fractionation in an aqueous medium, showing that $\mathrm{C}-\mathrm{O}$ and $\mathrm{C}-\mathrm{O}-\mathrm{C}$ groups are present both on the outer surface of the polycrystals and on the inner interphase boundaries. The absorption band at $1100-1110 \mathrm{~cm}^{-1}$ has different intensities in the polycrystals of the different size-fractions and is a maximum for the PCD-4 polycrystal fraction. Spectra $1-4$ in Fig. 6(a) are normalized to the intensity of the peak at $1800 \mathrm{~cm}^{-1}$, which is associated with vibrations of the carbonyl groups that result from chemical processing on the outer surface of the polycrystals-i.e., those exposed only to external agents. This means that the carbonyl groups enter into the composition of the carboxyl or anhydride groups on the surface of the polycrystals, while the $\mathrm{C}-\mathrm{O}-\mathrm{C}$ groups are fragments of a different type of atomic structures associated with the internal and external interfaces (by analogy with the model of [19] for DND aggregates). The internal interfaces that separate the crystallites in the polycrystals can also contain island-type graphite-like interlayers of atomic thickness. The intensity ratio of the absorption peaks at 1110 and $1800 \mathrm{~cm}^{-1}\left(I_{1110} / I_{1800}\right)$ is approximately proportional to the size of the polycrystalline particles in the interval from 25 to $100 \mathrm{~nm}$. This means that $\mathrm{C}-\mathrm{O}$ and $\mathrm{C}-\mathrm{O}-\mathrm{C}$ groups are present in the inner volume of the polycrystalline particles on the intercrystalline interfaces. The presence of covalent $\mathrm{C}-\mathrm{O} / \mathrm{C}-\mathrm{O}-\mathrm{C}$ bonds at the grain 
(a)

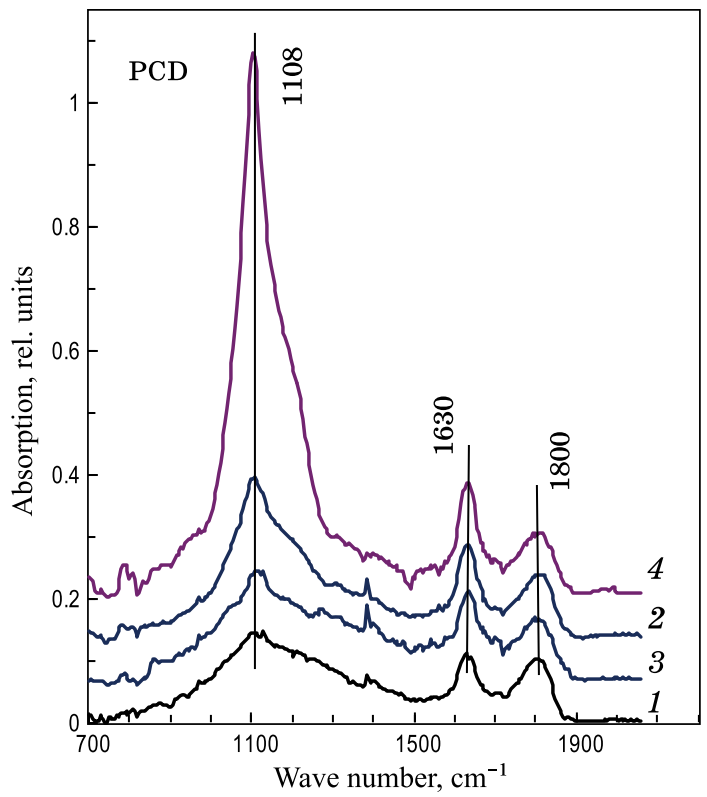

(b)

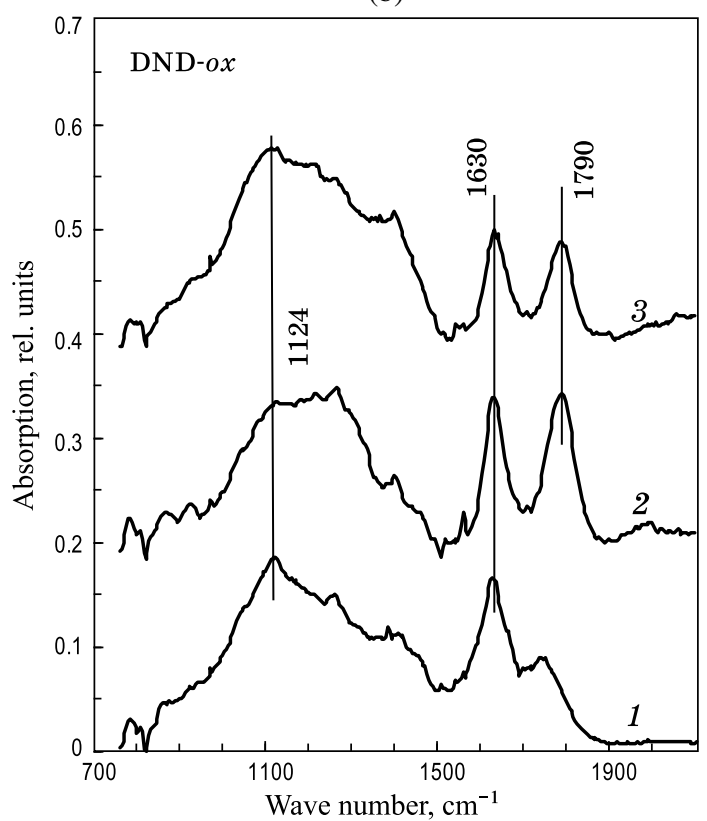

Fig. 6. Infrared absorption spectra of (a) the smallest size-fractions of polycrystalline diamond, formed in mixtures of acids in an autoclave and (b) detonation diamond DND-ox heat-treated in air. (a) shows the following size-fractions: 1-PCD-1, 2-PCD-2, 3-PCD-3, 4PCD-4. (b) shows the following process temperatures in ${ }^{\circ} \mathrm{C}: 1-$ 350, 2-430, 3-520. For simplicity, the two groups of spectra are shifted vertically by (a) $0.07,0.14$, and 0.21 and (b) 0.2 and 0.4 scale units, respectively.

boundaries and interfaces between the crystallites, apparently densely sintered and bonded to each other during the shock-wave synthesis, explains the excellent stability of the polycrystalline particles of PCD against chemical processing in acids at temperatures all the way to $190^{\circ} \mathrm{C}$. It is assumed here that the oxidizing chemical agent does not penetrate inside the polycrystals along the intergrain boundaries and interfaces between the crystallites. At the same time, it is impossible to completely rule out that the absorption peak at $1110 \mathrm{~cm}^{-1}$ is associated with valence vibrations in $\mathrm{C}-\mathrm{N}$ groups. The absorption band with a peak at $1110 \mathrm{~cm}^{-1}$ is relatively wide and contains a high-frequency wing (rise), which is obviously formed as a result of a second, nearby band (contour) alongside, with center at $1200 \mathrm{~cm}^{-1}$ [Fig. 6(a), curve 4]. Additional Gaussian-type contours that strongly overlap with the bands at 1100 and $1200 \mathrm{~cm}^{-1}$ may lie between them in the range from 1100 to $1200 \mathrm{~cm}^{-1}$. As is well known, substitutional nitrogen defects cause an absorption line at $1130 \mathrm{~cm}^{-1}$ in bulk nitrogen-doped diamond crystals ( $\mathrm{C}$ defects) in the concentration range from 100 to $2000 \mathrm{ppm}$ or even higher [20]. However, in the case of the internal intergrain boundaries (interfaces) between the crystallites in the polycrystals, a significantly greater amount of atomic nitrogen can be contained on the boundaries of these crystallites, saturating all the broken bonds that are present there and participating in the structure of the different configurations of strong $\mathrm{C}-\mathrm{N}-\mathrm{C}$ links between the crystallites. Such a possibility cannot be ruled out, considering that nitrogen was incorporated into the lattice at the synthesis stage when $\mathrm{NV}^{(-)}$centers were formed. As is well known, $\mathrm{Si}-\mathrm{O}$ bonds can also give strong absorption peaks at $1100 \mathrm{~cm}^{-1}$ if an amorphous $\mathrm{SiO}_{x}$ phase is present around the diamond nanocrystals [21]. However, the amount of silicon available in the sample (it is proven in [15] that silicon and $\mathrm{SiV}$ defects are present) is clearly insufficient in our case to explain the presence of strong absorption bands at $1100 \mathrm{~cm}^{-1}$ in the polycrystalline diamonds of the small size-fractions (PCD-1PCD-4). The silicon concentration is less than $100-140 \mathrm{ppm}$ in all the size-fractions of factory-processed polycrystalline diamond and is already less than 10-12 ppm in chemically purified powders.

The IR absorption spectrum of the reference DND sample of interest (DND-ox) is shown for comparison in Fig. 6(b) (spectrum 1). It is very similar in appearance to the IR absorption spectrum of the PCD-1 polycrystalline diamond sample considered above. The combined band at $1630 \mathrm{~cm}^{-1}$ is associated with valence vibrations of the hydroxyl groups of water molecules sorbed on the surface of the DND particles or in the potassium bromide matrix and is not eliminated from it at the stage of pressing the mixture. The spectrum contains a wide structureless band of triangular shape in the interval $850-1500 \mathrm{~cm}^{-1}$, which in the $1000-1300 \mathrm{~cm}^{-1}$ region is also predominantly associated with absorption at the vibrations of the $\mathrm{C}-\mathrm{O}$ and $\mathrm{C}-\mathrm{O}-\mathrm{C}$ groups that are present on the surface of the particles and a small band of Gaussian shape in the neighborhood of $1740 \mathrm{~cm}^{-1}$, associated with absorption at the valence vibrations of carbonyl groups. The given absorption is characteristic of DND processed in acids and was mentioned earlier in [22] and [23]. In the wide $850-1500 \mathrm{~cm}^{-1}$ band, at least five overlapping absorption bands of Gaussian shape can be distinguished at 970,1050,1120,1250, and $1400 \mathrm{~cm}^{-1}$. Three of these peaks (at 1050, 1120, and $1250 \mathrm{~cm}^{-1}$ ) are apparently fundamental characteristics of the oxidized surface of diamond and were observed earlier in the absorption spectra of variously processed detonation nanodiamonds [22,23]. A peak 
with maximum at $1100-1130 \mathrm{~cm}^{-1}$ was pointed out in virtually all papers on the IR spectroscopy of DND.

Figure 6(b) also shows the absorption spectra of the DNDox reference sample subjected to heat treatment in air. Spectra 2 and 3 in Fig. 6(b) are evidence of substantial changes in the composition of the functional groups on the surface of isolated DND-ox nanoparticles or aggregates as a function of the processing temperature in air. The absorption zone at $1100-1130 \mathrm{~cm}^{-1}$ is also present on the spectrum of DNDox, as it is in PCD-1 and is evidence of the presence of $\mathrm{C}-\mathrm{O}$ and $\mathrm{C}-\mathrm{O}-\mathrm{C}$ groups both on the outer surface of the DND particles and on the inner boundaries in DND aggregates with a mean size of $30 \mathrm{~nm}$. In the interval of treatment temperatures from $350^{\circ} \mathrm{C}$ to $520^{\circ} \mathrm{C}$, the absorption spectra of DND-ox (spectra 2 and 3) includes an absorption band at 1780-1790 $\mathrm{cm}^{-1}$ from carbonyl groups. It forms when diamond undergoes dry or moist surface oxidation in air. The $1780-1790 \mathrm{~cm}^{-1}$ absorption peak from carbonyl groups is already substantially decreased at $t \geq 550^{\circ} \mathrm{C}$ (by a large factor; the spectrum is not shown on the figure), and this is associated with gas-phase etching of the surface and ablation of the material. The latter is most distinctly seen and has the greatest intensity at temperatures of $430^{\circ} \mathrm{C}-520^{\circ} \mathrm{C}$ [Fig. 6(b), spectra 2 and 3], and this is evidence that carbonyl groups are prevalent on the surface in this range of heat treatment. The highest luminescence intensity of the $\mathrm{NV}^{(-)}$centers is demonstrated by DND particles subjected to such treatment, which are optimal for applied uses regardless of their morphological statusisolated $5-\mathrm{nm}$ particles or aggregates with a mean size of $30-35 \mathrm{~nm}$. The changes that occur when $t \geq 550^{\circ} \mathrm{C}$ are associated with surface etching of the particles, ablation of the material by volatile reaction products of oxidation, and the appearance of a family of functional groups of another composition on the DND surface [24].

\section{CONCLUSION}

It has thus been demonstrated that the EPR method is extremely effective for estimating the concentration of $\mathrm{NV}^{(-)}$ centers in synthetic fluorescent nanodiamonds, where these centers appeared at the synthesis stage or were later specially created. For particles in the size range up to $7 \mathrm{~nm}$, the greatest concentration of $\mathrm{NV}^{(-)}$centers $(2.7 \mathrm{ppm})$ is reached in detonation diamonds; however, because of the total defect content of the crystal lattice of particles of DND and its surface, the luminescence intensity is low because of different types of quenching effects. Nevertheless, each 30-35-nm aggregate consisting of densely sintered DND particles contains from two to five $\mathrm{NV}^{(-)}$centers and is optically recorded individually. Dynamically sythesized polycrystalline diamond particles also display $\mathrm{NV}^{(-)}$luminescence. Their $\mathrm{NV}^{(-)}$concentration is less than that of DND. However, the concentration of $\mathrm{NV}^{(-)}$centers and the luminescence intensity from the $\mathrm{NV}^{(-)}$centers are greatest in polycrystalline diamonds whose aggregates have a mean size of $180 \mathrm{~nm}$. The effective concentration of $\mathrm{NV}^{(-)}$ centers in smaller polycrystalline aggregates is lower because of deactivation effects of the centers by surface effects or because they break down in the mechanically damaged surface layer. Specially irradiated and annealed synthetic fluorescent
HPHT Ib diamonds demonstrate a high concentration of $\mathrm{NV}^{(-)}$centers (up to 4-5 ppm) along with high quality of the crystal lattice; this is especially important for obtaining high-intensity fluorescence radiation from submicrometer $(0.1 \mu \mathrm{m})$ particles and is best-adapted to technical and other applications. Fluorescent DND and FND particles can be used to create subsurface marks and markers in plastics, biopolymers, and polymeric composites that weakly absorb optical radiation in the 600-750 $\mathrm{nm}$ spectral range, especially in those regions where long-lived stability and thermal resistance of the luminescence centers is actually present under conditions of a chemically aggressive atmosphere or UV radiation.

Funding. Russian Science Foundation (RSF) (14-1300795); Japan Society for the Promotion of Science (JSPS) (L17526); Russian Foundation for Basic Research (RFBR) (17-52-50004 YaF_a).

Acknowledgment. The authors are grateful to Professor Takuya Hayashi from Shinshu University (Nagano, Japan) for help in obtaining images of particles by high-resolution transmission electron microscopy. V. Yu. Osipov is grateful to Hosei University (Tokyo, Japan) for collaboration.

\section{REFERENCES}

1. A. Vul' and O. Shenderova, eds., Detonation Nanodiamonds: Science and Applications (Pan Stanford, Singapore, 2014).

2. V. N. Mochalin, O. Shenderova, D. Ho, and Y. Gogotsi, "The properties and applications of nanodiamonds," Nat. Nanotechnol. 7(1), 11 (2012).

3. O. Shenderova and G. McGuire, "Science and engineering of nanodiamond particle surfaces for biological applications," Biointerphases 10, 030802 (2015).

4. R. M. Hazen, "The new diamond makers: diamonds by explosion," in The Diamond Makers (Cambridge University Press, Cambridge, 1999), Chap. 11, pp. 190-198.

5. J. Beard, "Explosive mixtures," New Scientist (1637), 43-47 (1988).

6. L. N. Teasley, N. F. Bailey, and O. R. Bergmann, "Micropolycrystalline diamond by shock synthesis: advances in performance and property characterization," Proc. Electrochem. Soc. 97-32, 48-57 (1998).

7. P. D. Ownby, "Nano $6 \mathrm{H}$ diamond polytype polycrystalline powder," NSTI-Nanotech. 3, 210 (2004).

8. A. I. Shames, V. Yu. Osipov, H. J. von Bardeleben, and A. Ya. Vul', "Spin $\mathrm{S}=1$ centers: a universal type of paramagnetic defects in nanodiamonds of dynamic synthesis," J. Phys. Condens. Matter 24(22), 225302 (2012).

9. J. H. N. Loubser and J. A. van Wyk, "Electron spin resonance in the study of diamond," Rep. Prog. Phys. 41, 1201 (1978).

10. M. W. Doherty, N. B. Manson, P. Delaney, F. Jelezko, J. Wrachtrup, and L. C. Hollenberg, "The nitrogen-vacancy colour centre in diamond," Phys. Rep. 528(1), 1 (2013).

11. A. I. Shames, V. Yu. Osipov, H. J. von Bardeleben, J.-P. Boudou, F. Treussart, and A. Ya. Vul', "Native and induced triplet nitrogenvacancy centers in nano- and microdiamonds: half-field electron paramagnetic resonance fingerprint," Appl. Phys. Lett. 104, 063107 (2014).

12. A. I. Shames, V. Yu. Osipov, J. P. Boudou, A. M. Panich, H. J. von Bardeleben, F. Treussart, and A. Ya. Vul', "Magnetic resonance tracking of fluorescent nanodiamond fabrication," J. Phys. D: Appl. Phys. 48(15), 155302 (2015).

13. V. Yu. Osipov, A. I. Shames, T. Enoki, K. Takai, M. V. Baidakova, and A. Ya. Vul, "Paramagnetic defects and exchange coupled spins in pristine ultrananocrystalline diamonds," Diamond Relat. Mater. 16(12), 2035 (2007). 
14. A. I. Shames, D. Mogilyansky, A. M. Panich, N. A. Sergeev, M. Olszewski, J.-P. Boudou, and V. Yu. Osipov, "XRD, NMR, and EPR study of polycrystalline micro- and nanodiamonds prepared by a shock wave compression method," Phys. Status Solidi A 212(11), 2400 (2015).

15. K. V. Bogdanov, V. Yu. Osipov, M. V. Zhukovskaya, C. Jentgens, F. Treussart, T. Hayashi, K. Takai, A. V. Fedorov, and A. V. Baranov, "Size-dependent Raman and SiV-center luminescence in polycrystalline nanodiamonds produced by shock wave synthesis," RSC Adv. 6 , 51783 (2016).

16. A. I. Shames, V. Yu. Osipov, K. V. Bogdanov, A. V. Baranov, M. V. Zhukovskaya, A. Dalis, S. S. Vagarali, and A. Rampersaud, "Does progressive nitrogen doping intensify negatively charged nitrogen vacancy emission from e-beam-irradiated Ib type high-pressure-hightemperature diamonds?" J. Phys. Chem. C 121(9), 5232 (2017).

17. K. lizuka, Y. Furukawa, and R. Oshima, "Analysis of binding state of heat treated diamond powder," Am. J. Chem. Mater. Sci. 1(1), 7 (2014).

18. N. B. Colthup, L. H. Daly, and S. E. Wiberley, Introduction to Infrared and Raman Spectroscopy (Academic Press, New York, 1964).
19. K. Xu and Q. Xue, "New method for deaggregation of nanodiamond from explosive detonation: graphitization-oxidation method," Phys. Solid State 46(4), 649 (2004) [Fiz. Tverd. Tela 46(4), 633 (2004)].

20. A. M. Zaitsev, Optical Properties of Diamond: A Data Handbook (Springer-Verlag, Berlin, 2001).

21. I. Rehor, J. Slegerova, J. Kucka, V. Proks, V. Petrakova, M.-P. Adam, F. Treussart, S. Turner, S. Bals, P. Sacha, M. Ledvina, A. M. Wen, N. F. Steinmetz, and P. Cigler, "Fluorescent nanodiamonds: fluorescent nanodiamonds embedded in biocompatible translucent shells," Small 10(6), 1106 (2014).

22. I. I. Kulakova, "Surface chemistry of nanodiamonds," Phys. Solid State 46(4), 636 (2004) [Fiz. Tverd. Tela 46(4), 621 (2004)].

23. Y. W. Zhu, X. Q. Shen, B. C. Wang, X. Y. Xu, and Z. J. Feng, "Chemical mechanical modification of nanodiamond in aqueous system," Phys. Solid State 46(4), 681 (2004) [Fiz. Tverd. Tela 46(4), 665 (2004)].

24. S. Osswald, G. Yushin, V. Mochalin, S. O. Kucheyev, and Y. Gogotsi, "Control of $\mathrm{sp}^{2} / \mathrm{sp}^{3}$ carbon ratio and surface chemistry of nanodiamond powders by selective oxidation in air," J. Am. Chem. Soc. 128(35), 11635 (2006). 